\title{
Voltage-induced precessional switching at zero-bias magnetic field in a conically magnetized free layer
}

\author{
R. Matsumoto:* T. Nozaki, S. Yuasa, and H. Imamurdt \\ ${ }^{1}$ National Institute of Advanced Industrial Science and Technology (AIST), \\ Spintronics Research Center, Tsukuba, Ibaraki 305-8568, Japan
}

(Dated: August 12, 2018)

\begin{abstract}
Voltage-induced magnetization dynamics in a conically magnetized free layer with an elliptic cylinder shape is theoretically studied on the basis of the macrospin model. It is found that an application of voltage pulse can induce the precessional switching of magnetization even at zero-bias magnetic field, which is of substantial importance for device applications such as voltage-controlled nonvolatile memory. Analytical expressions of the conditions for precessional switching are derived.
\end{abstract}

* rie-matsumoto@aist.go.jp

$\dagger$ h-imamura@aist.go.jp 


\section{INTRODUCTION}

Voltage control of magnetic anisotropy (VCMA) in a ferromagnetic metal layer is a promising technology for the low-power writing in magnetoresistive random-access mem-

ories (MRAMs) [1 14] compared to the writing with spin-transfer torque (STT) 15 17]. In the magnetic tunnel junctions with perpendicular magnetization (p-MTJs) which have been the mainstream technology for STT-MRAMs, voltage-driven writing-i.e., magnetization switching-has been demonstrated by an application of a bias voltage with an appropriate pulse width under a bias magnetic field having an in-plane (IP) component [11 14$]$.

For practical applications, writing at zero-bias magnetic field is desirable to simplify the device structure and reduce the fabrication cost. It is known that in magnetoresistive devices the shape anisotropy field can act as a bias magnetic field in some situations. The IP shape anisotropy field is obtained by microfabricating a ferromagnetic film into an elliptic cylinder shape. In the case of a perpendicularly magnetized free layer, however, the IP shape anisotropy field cannot move its magnetization from the perpendicular direction because the IP shape anisotropy field is proportional to the IP component of the magnetization. It is necessary to tilt the magnetization from the perpendicular direction to perform the precessional switching of the magnetization around the IP shape anisotropy field. 

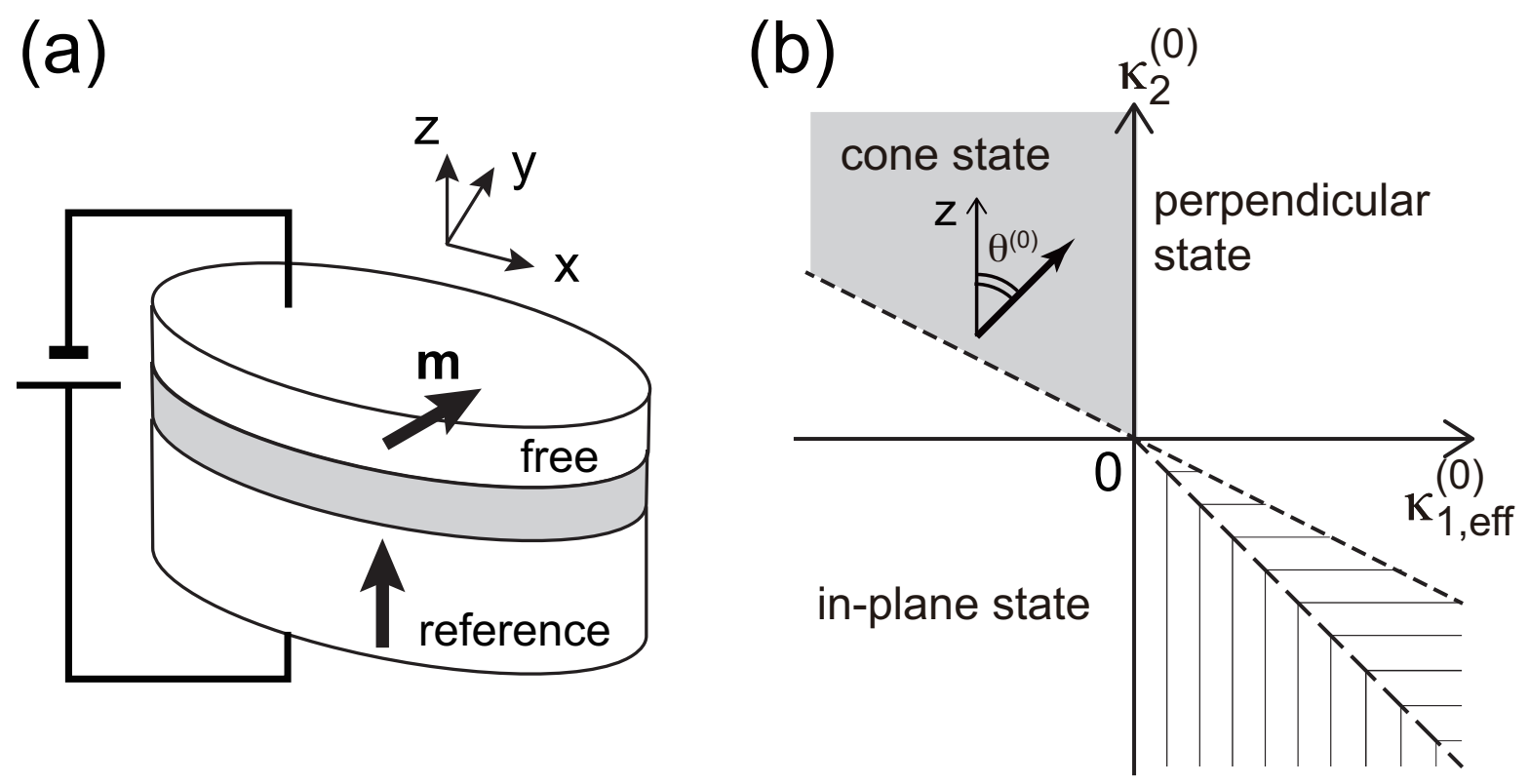

FIG. 1. (a) Magnetic tunnel junction with elliptic cylinder shape and definitions of Cartesian coordinates $(x, y, z)$. The $x$ axis is parallel to the major axis of the ellipse. (b) Phase diagram of magnetic film with uniaxial anisotropy constants $\kappa_{1 \text {,eff }}^{(0)}$ and $\kappa_{2}^{(0)}$ at equilibrium state (redrawn from Ref. 18). The shaded area represents the cone-state phase, where the film is conically magnetized with equilibrium polar angle, $\theta^{(0)}$. The bistable regions are hatched.

The titled magnetization state can be obtained by using a conically magnetized film as a free layer [19 21]. The conically magnetized state, which is stabilized by the competition between the first- and second-order magnetic anisotropy energies, has been experimentally observed in double-layer and multilayer systems [22, 23]. Recently, the VCMA effect [24] in addition to the conically magnetized state [25 27] has been observed in a Co-Fe-B thin film which is commonly used as a free layer in an MRAM.

In this paper, the voltage-driven precessional switching in a conically magnetized free layer with an elliptic cylinder shape is analyzed. Analytical expressions of the conditions for precessional switching at zero-bias magnetic field are derived.

\section{MODEL}

The system we consider is schematically shown in Fig. 11(a). The lateral size of the nanopillar is assumed to be so small that the magnetization dynamics can be described by 
the macrospin model. The direction of the magnetization in the free layer is represented by the unit vector $\boldsymbol{m}=\left(m_{x}, m_{y}, m_{z}\right)=(\sin \theta \cos \phi, \sin \theta \sin \phi, \cos \theta)$, where $\theta$ and $\phi$ are the polar and azimuthal angles of $\boldsymbol{m}$. The magnetization in the reference layer is fixed to align in the positive $z$ direction.

The energy density of the free layer is given by [28]

$$
\begin{aligned}
\mathcal{E}\left(m_{x}, m_{y}, m_{z}\right)= & \frac{1}{2} \mu_{0} M_{\mathrm{s}}^{2}\left(N_{x} m_{x}^{2}+N_{y} m_{y}^{2}+N_{z} m_{z}^{2}\right) \\
& +K_{\mathrm{u} 1}\left(1-m_{z}^{2}\right)+K_{\mathrm{u} 2}\left(1-m_{z}^{2}\right)^{2}
\end{aligned}
$$

where $K_{\mathrm{u} 1}$ and $K_{\mathrm{u} 2}$ are the first- and second-order anisotropy constants, respectively. The values of $K_{\mathrm{u} 1}$ and $K_{\mathrm{u} 2}$ can be varied by application of a bias voltage, $V$, through the VCMA effect. The demagnetization coefficients, $N_{x}, N_{y}$ and $N_{z}$ are assumed to satisfy $N_{z} \gg N_{y}>N_{x} . \mu_{0}$ is the vacuum permeability, and $M_{\mathrm{s}}$ is the saturation magnetization of the free layer. IP shape anisotropy field, $H_{\mathrm{k}}$, is given by $H_{\mathrm{k}}=M_{\mathrm{s}}\left(N_{y}-N_{x}\right)$ [29]. Normalization by $\mu_{0} M_{\mathrm{s}}^{2}$ leads to the dimensionless energy density defined as [28]

$$
\begin{aligned}
\varepsilon\left(m_{x}, m_{y}, m_{z}\right)= & \frac{1}{2}\left(N_{x} m_{x}^{2}+N_{y} m_{y}^{2}+N_{z} m_{z}^{2}\right) \\
& +\kappa_{1}\left(1-m_{z}^{2}\right)+\kappa_{2}\left(1-m_{z}^{2}\right)^{2}
\end{aligned}
$$

where $\varepsilon=\mathcal{E} /\left(\mu_{0} M_{\mathrm{s}}^{2}\right), \kappa_{1}=K_{\mathrm{u} 1} /\left(\mu_{0} M_{\mathrm{s}}^{2}\right)$ and $\kappa_{2}=K_{\mathrm{u} 2} /\left(\mu_{0} M_{\mathrm{s}}^{2}\right)$.

Before analyzing the switching conditions, let us show the basic properties of the equilibrium magnetization state at $V=0$, which is the initial state of magnetization switching. The direction of the magnetization of the initial state, $\boldsymbol{m}^{(0)}$, is obtained by minimizing the energy density at $V=0$ as [18]

$$
m_{z}^{(0)}= \pm \sqrt{1+\frac{\kappa_{1, \mathrm{eff}}^{(0)}}{2 \kappa_{2}^{(0)}}}
$$

$m_{x}^{(0)}= \pm \sqrt{1-\left(m_{z}^{(0)}\right)^{2}}$, and $m_{y}^{(0)}=0$. Throughout the paper, the superscript $(0)$ indicates the quantities at $V=0$. Without loss of generality, the initial state is assumed to satisfy $m_{x}^{(0)}>0$ and $m_{z}^{(0)}>0$. The effective first-order anisotropy constant, $\kappa_{1, \text { eff }}$, is defined as $\kappa_{1, \text { eff }}=\kappa_{1}-(1 / 2)\left(N_{z}-N_{x}\right)$. Since we are interested in the voltage-induced switching of a conically magnetized free layer, we concentrate on the cone-state region indicated by the shaded region in Fig. 1(b), where $\kappa_{1, \text { eff }}^{(0)}<0$ and $\kappa_{2}^{(0)}>-(1 / 2) \kappa_{1, \text { eff }}^{(0)}$. 
The retention time of an MRAM is quantified by the thermal stability factor, $\Delta^{(0)}$, which is defined by the ratio of the energy barrier to the thermal energy, $k_{\mathrm{B}} T$, as

$$
\Delta^{(0)}=\frac{\left[\mathcal{E}^{(0)}(1,0,0)-\mathcal{E}^{(0)}\left(m_{x}^{(0)}, 0, m_{z}^{(0)}\right)\right] V_{\mathrm{F}}}{k_{\mathrm{B}} T},
$$

where $V_{\mathrm{F}}$ represents the volume of the free layer, $k_{\mathrm{B}}$ is the Boltzmann constant and $T$ is temperature. The volume of the free layer is expressed as $V_{\mathrm{F}}=\pi r_{x} r_{y} t_{\mathrm{F}}$, where $r_{x}\left(r_{y}\right)$ is half the length of the major (minor) axis of an ellipse, and $t_{\mathrm{F}}$ is thickness of the free layer. The thermal stability factor at room temperature should be more than 60 for the retention time over 10 years.

Although our analysis can be applied to a quite general situation, it is difficult to understand its benefit without showing specific examples. The following parameters are assumed: $M_{\mathrm{S}}=1400 \mathrm{kA} / \mathrm{m}, r_{x}=50 \mathrm{~nm}, r_{y}=20 \mathrm{~nm}, t_{\mathrm{F}}=1 \mathrm{~nm}, N_{x}=0.0122, N_{y}=0.0443$, $N_{z}=0.9435$ [30], $H_{\mathrm{k}}=566$ Oe, $K_{1, \text { eff }}^{(0)}=-80 \mathrm{~kJ} / \mathrm{m}^{3}, K_{\mathrm{u} 1}^{(0)}=1067 \mathrm{~kJ} / \mathrm{m}^{3}, K_{\mathrm{u} 2}^{(0)}=150$ $\mathrm{kJ} / \mathrm{m}^{3}, m_{z}^{(0)}=0.856, \Delta^{(0)}$ at $T=300 \mathrm{~K}$ is 61.2 . The equilibrium polar angle is $\theta^{(0)}=31.1^{\circ}$, which corresponds to a $20 \%$ reduction of the magnetoresistance ratio from the case of the perpendicularly magnetized free layer. The direction of $\boldsymbol{m}^{(0)}$ is indicated by the open circle in Fig. 2(a) for the contour plot of $\varepsilon^{(0)}$. 
(a)

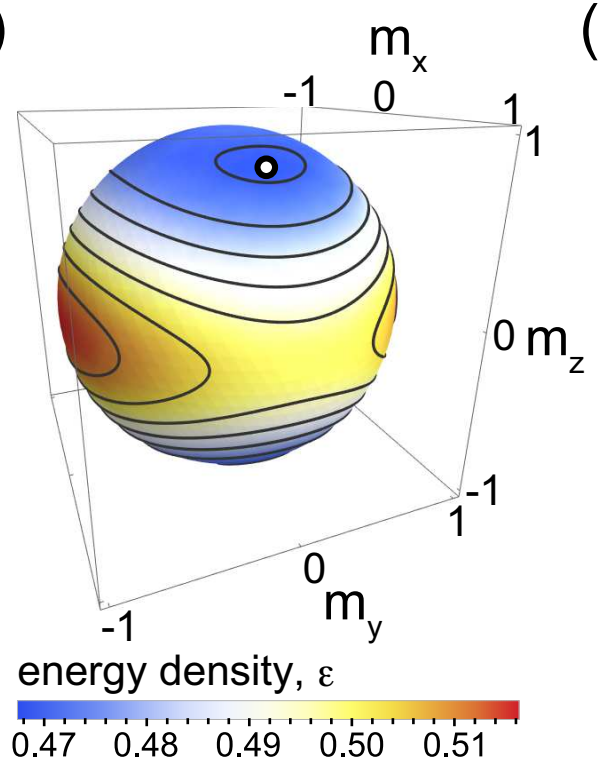

(c)

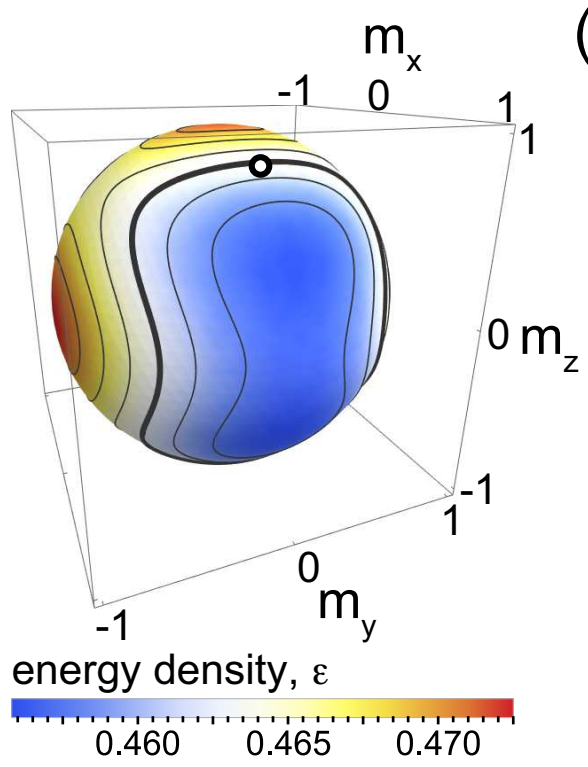

(b)

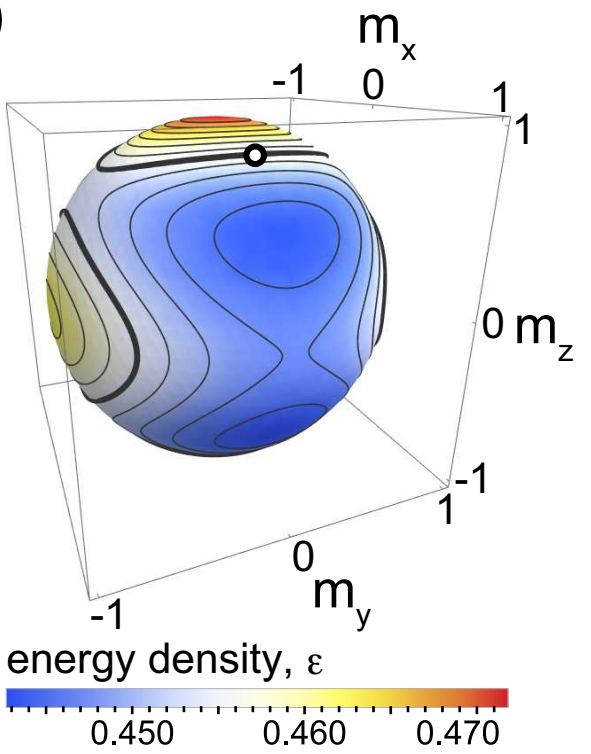

(d)

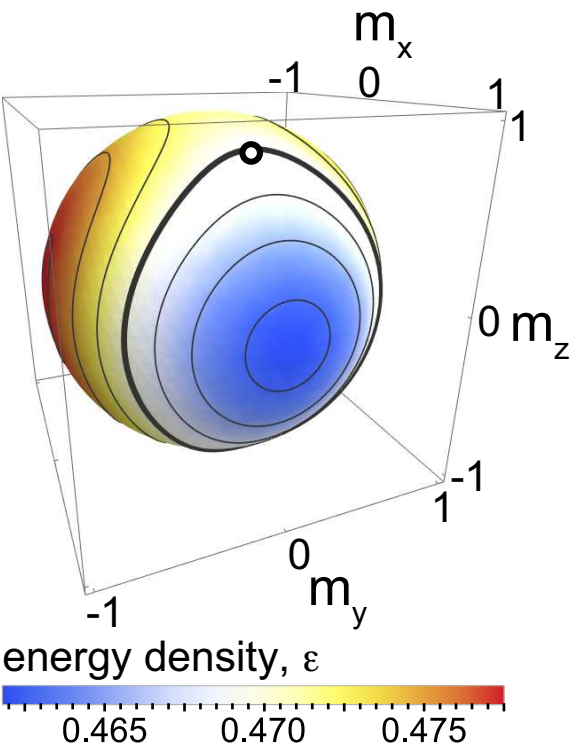

FIG. 2. (a) Energy-density contour plot of Eq. (2) for the initial state, $\boldsymbol{m}^{(0)}$. The direction of $\boldsymbol{m}^{(0)}$ is indicated by the open circle. (b) Typical example of the energy-density contour plot of Eq. (2) in the horizontally hatched region in Fig. 3. The anisotropy constants are assumed to be $\left(\kappa_{1, \text { eff }}, \kappa_{2}\right)=(-0.080,0.055)$. The open circle indicates $\boldsymbol{m}^{(0)}$. Thick solid curves represent the contour having the same energy density as $\varepsilon\left(\boldsymbol{m}^{(0)}\right)$. (c) The same plot in the vertically hatched region in Fig. 3, The anisotropy constants are assumed to be $\left(\kappa_{1, \text { eff }}, \kappa_{2}\right)=(-0.040,0.025)$. (d) The same plot in the diagonally hatched region in Fig. 3. The anisotropy constants are assumed to be $\left(\kappa_{1, \mathrm{eff}}, \kappa_{2}\right)=(-0.005,-0.005)$. 


\section{RESULTS}

In voltage-driven precessional switching, only a half period of precessional motion is used to switch the magnetization. Thanks to the smallness of the damping constant of the free layer, the trajectory of the initial half period of precession is well represented by an energydensity contour including $\boldsymbol{m}^{(0)}$ on the Bloch sphere (see Appendix A). Application of a bias voltage modifies the anisotropy constants, destabilizes the initial state, and induces the precessional motion. If the trajectory or energy-density contour starting from $\boldsymbol{m}^{(0)}$ crosses the equator of the Bloch sphere, i.e., $m_{z}=0$, the magnetization can be switched by turning off the voltage after a half period of precession. It is important to find the values of $\kappa_{1 \text {,eff }}$ and $\kappa_{2}$ which enable precessional switching, i.e., the conditions for precessional switching.

The energy-density contour having the same energy density as $\varepsilon\left(\boldsymbol{m}^{(0)}\right)$ is expressed as

$$
\varepsilon(\boldsymbol{m})=\varepsilon\left(\boldsymbol{m}^{(0)}\right)
$$

The substitution of $m_{z}=0$ into Eq. (5) yields

$$
\frac{1}{2}\left(N_{x}-N_{y}\right) m_{x}^{2}+\frac{1}{2} N_{y}+\kappa_{1}+\kappa_{2}=\varepsilon\left(\boldsymbol{m}^{(0)}\right)
$$

Requiring $0<m_{x}^{2}<1$, one obtains the following inequality

$$
-\xi \kappa_{1, \mathrm{eff}}-\eta<\kappa_{2}<-\xi \kappa_{1, \mathrm{eff}}
$$

where

$$
\xi=\frac{1}{2-\left(m_{z}^{(0)}\right)^{2}}, \eta=\frac{\xi\left(N_{y}-N_{x}\right)}{2\left(m_{z}^{(0)}\right)^{2}}
$$

Equation (7) represents the condition for the energy-density contour to cross the equator. The upper and lower boundaries of Eq. (7) are indicated by thin dotted lines in Fig. 3. 


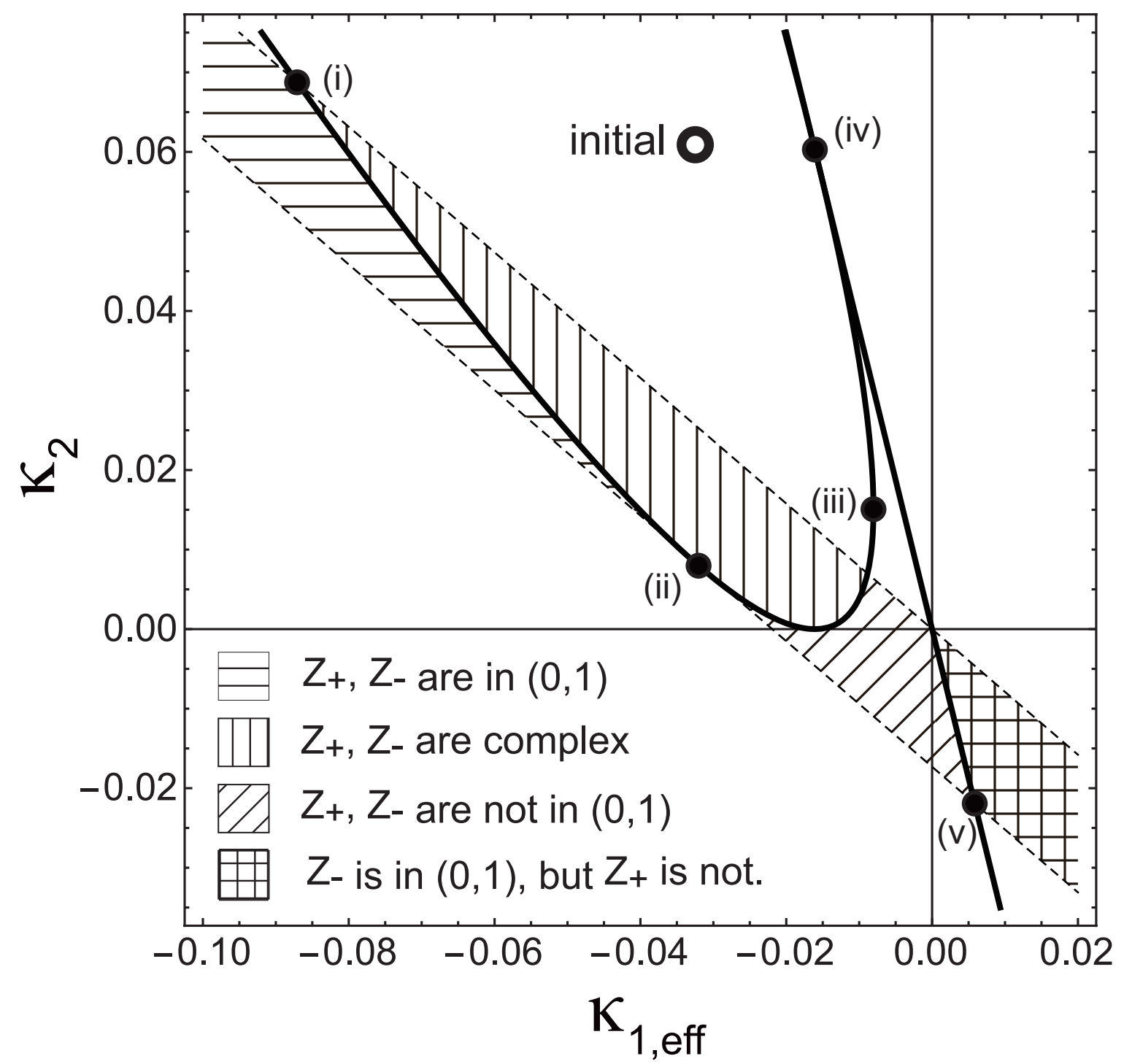

FIG. 3. Classification of the values of $Z_{+}$and $Z_{-}$in the $\kappa_{1 \text {,eff }}$ and $\kappa_{2}$ plane. The thin dotted lines represent the upper and lower boundaries of Eq. (7). The thick solid curves, which are smoothly connected with each other at point (iii), represent Eqs. (13) and (17), respectively. The thick solid line represents Eq. (19). In the horizontally hatched region, both $Z_{+}$and $Z_{-}$are in the region $(0,1)$. In the vertically hatched region, both $Z_{+}$and $Z_{-}$are complex numbers. In the diagonally hatched region, both $Z_{+}$and $Z_{-}$are real numbers but not in the region $(0,1)$. In the crosshatched region, both $Z_{+}$and $Z_{-}$are real numbers, but only $Z_{-}$is in the region $(0,1)$. The point corresponding to the initial state is represented by the open circle. The values of $\kappa_{1, \text { eff }}$ at the points indicated by the solid circles (i) - (v) are given in the text.

There are two kinds of energy-density contour crossing the equator. One crosses the 
latitude with $m_{y}=0$ and surrounds the $m_{x}$ axis. The other crosses the latitude with $m_{x}=0$ and surrounds the $m_{y}$ axis. The energy density $\varepsilon(\boldsymbol{m})$ has symmetry under a sign change of $m_{x}$. Even if $\boldsymbol{m}^{(0)}$ is located on the energy-density contour surrounding the $m_{y}$ axis, it connects the initial state $\left(m_{x}^{(0)}, m_{y}^{(0)}, m_{z}^{(0)}\right)$ with $\left(-m_{x}^{(0)}, m_{y}^{(0)}, m_{z}^{(0)}\right)$ and does not result in switching. The region of $\left(\kappa_{1, \text { eff }}, \kappa_{2}\right)$ corresponding to such trajectories should be excluded in the region defined by Eq. (17).

The solutions of Eq. (6) are given by

$$
\tilde{m}_{x}= \pm \sqrt{\frac{2}{N_{y}-N_{x}}\left(\frac{1}{2} N_{y}+\kappa_{1}+\kappa_{2}-\varepsilon\left(\boldsymbol{m}^{(0)}\right)\right)} .
$$

The energy-density contour crosses the equator, at most, at four points: $\left( \pm \tilde{m}_{x}, \pm \tilde{m}_{y}, 0\right)$, where $\tilde{m}_{y}=\sqrt{1-\tilde{m}_{x}^{2}}$. Therefore, the energy-density contour with $\varepsilon\left(\boldsymbol{m}^{(0)}\right)$ corresponding to the precession around the $m_{x}$ axis does not coexist with that corresponding to the precession around the $m_{y}$ axis.

The value of $m_{z}$ at which energy-density contour crosses the latitude with $m_{x}=0$ is obtained as follows. Substitution of $m_{x}=0$ into Eq. (5) leads to

$$
\begin{aligned}
& \kappa_{2} Z^{2}+\left[\frac{1}{2}\left(N_{z}-N_{y}\right)-\kappa_{1}-2 \kappa_{2}\right] Z \\
& +\frac{1}{2} N_{y}+\kappa_{1}+\kappa_{2}=\varepsilon\left(\boldsymbol{m}^{(0)}\right),
\end{aligned}
$$

where $Z=m_{z}^{2}$. The solutions of Eq. (10) are obtained as

$$
Z_{ \pm}=\frac{-\left[\frac{1}{2}\left(N_{z}-N_{y}\right)-\kappa_{1}-2 \kappa_{2}\right] \pm \sqrt{D}}{2 \kappa_{2}}
$$

where

$$
\begin{aligned}
D & =\left[\frac{1}{2}\left(N_{z}-N_{y}\right)-\kappa_{1}-2 \kappa_{2}\right]^{2} \\
& -4 \kappa_{2}\left[\frac{1}{2} N_{y}+\kappa_{1}+\kappa_{2}-\varepsilon\left(\boldsymbol{m}^{(0)}\right)\right] .
\end{aligned}
$$

The energy-density contour crosses the latitude with $m_{x}=0$ at $m_{z}= \pm \sqrt{Z_{ \pm}}$if $0<Z_{ \pm}<1$.

In the region defined by Eq. (7), the solutions are classified into four groups as shown in Fig. 3. In the horizontally hatched region both $Z_{+}$and $Z_{-}$are in $(0,1)$, which means that the energy-density contour crossing the equator also crosses the latitude with $m_{x}=0$. No energy-density contour corresponding to the precessional switching exists in this region as shown in Fig. 2(b). 
In the vertically hatched region, the solutions $Z_{ \pm}$are complex, which means that the energy-density contour crossing the equator does not cross the latitude with $m_{x}=0$ but surrounds the $m_{x}$ axis. Precessional switching is available, as shown in Fig. 2(c). In Fig. 2 (c), the anisotropy constants under a bias voltage are assumed to be $\left(\kappa_{1, \text { eff }}, \kappa_{2}\right)=(-0.040$, $0.025)$ which is $\left(K_{1, \mathrm{eff}}, K_{\mathrm{u} 2}\right)=(-98.5,61.6) \mathrm{kJ} / \mathrm{m}^{3}$ in SI units.

The anisotropy constants, for example, can be obtained by the application of $V=1 \mathrm{~V}$, i.e., the electric field $\left(V / t_{\mathrm{I}}\right)$ when the VCMA effect is $\eta_{1}=18.5 \mathrm{fJ} /(\mathrm{V} \mathrm{m})$ and $\eta_{2}=88.4$ $\mathrm{fJ} /(\mathrm{V} \mathrm{m})$. Here, $t_{\mathrm{I}}$ represents the thickness of the insulator layer sandwiched between the free and the reference layers, and $t_{\mathrm{I}}=1 \mathrm{~nm}$ is assumed. the linear bias-voltage dependence of $K_{1, \text { eff }}$ and $K_{\mathrm{u} 2}$ are assumed, and $\eta_{1}$ and $\eta_{2}$ represent the coefficient of the VCMA effect for $K_{1, \text { eff }}$ and $K_{\mathrm{u} 2}$. The anisotropy constants per unit area, $K_{1, \mathrm{eff}} t_{\mathrm{F}}$ and $K_{\mathrm{u} 2} t_{\mathrm{F}}$, are expressed as $K_{1, \text { eff }} t_{\mathrm{F}}=K_{1, \text { eff }}^{(0)} t_{\mathrm{F}}-\eta_{1}\left(V / t_{\mathrm{I}}\right)$ and $K_{\mathrm{u} 2} t_{\mathrm{F}}=K_{\mathrm{u} 2}^{(0)} t_{\mathrm{F}}-\eta_{2}\left(V / t_{\mathrm{I}}\right)$.

It should be noted that the precessional switching is available even if $K_{\mathrm{u} 2}$ is not changed by the bias voltage [31, 32], i.e., $\eta_{2}=0$. For example, $\left(\kappa_{1, \text { eff }}, \kappa_{2}\right)=(-0.080,0.061)$ is included in the vertically hatched region while $\left(\kappa_{1, \text { eff }}^{(0)}, \kappa_{2}^{(0)}\right)=(-0.032,0.061)$ in the initial state. In SI units, $\left(K_{1, \text { eff }}, K_{\mathrm{u} 2}\right)=(-197,150) \mathrm{kJ} / \mathrm{m}^{3}$, and it can be obtained at $V=1 \mathrm{~V}$ and $t_{\mathrm{I}}=1$ $\mathrm{nm}$, i.e., the electric field $\left(V / t_{\mathrm{I}}\right)$ of $1 \mathrm{~V} / \mathrm{nm}$ and $\eta_{1}=117 \mathrm{fJ} /(\mathrm{V} \mathrm{m})$. Such linear bias-voltage dependence with $\eta_{1} \sim 100 \mathrm{fJ} /(\mathrm{V} \mathrm{m})$ has been experimentally demonstrated for the VCMA effect due to the modulation of charge accumulation without charge trapping [33 35$]$.

In the diagonally hatched region, the solutions $Z_{ \pm}$are real but outside of $(0,1)$, which means that the energy-density contour crossing the equator does not cross the latitude with $m_{x}=0$, and precessional switching is available, as shown in Fig. 2(d).

The boundary among the horizontally hatched, vertically hatched, and diagonally hatched regions is given by

$$
\kappa_{2}=\frac{-\kappa_{1, \mathrm{eff}}-\sqrt{\Lambda}}{2\left(1-Z^{(0)}\right)}
$$

where $Z^{(0)}=\left(m_{z}^{(0)}\right)^{2}$ and

$$
\Lambda=-\kappa_{1, \mathrm{eff}}\left(N_{y}-N_{x}\right)-\frac{1}{4}\left(N_{y}-N_{x}\right)^{2} .
$$

This boundary is plotted by the solid curve in Fig. 3. The curve of Eq. (13) crosses the upper boundary of Eq. (7) at point (i), where

$$
\kappa_{1, \mathrm{eff}}^{(\mathrm{i})}=-\frac{\left(N_{y}-N_{x}\right)\left(2-Z^{(0)}\right)\left(2-Z^{(0)}+2 \sqrt{1-Z^{(0)}}\right)}{2\left(Z^{(0)}\right)^{2}} .
$$


The curve of Eq. (13) is tangential to the lower boundary of Eq. (7) at point (ii), where

$$
\kappa_{1, \mathrm{eff}}^{(\mathrm{ii})}=-\frac{\left(N_{y}-N_{x}\right)\left[2-2 Z^{(0)}+\left(Z^{(0)}\right)^{2}\right]}{2\left(Z^{(0)}\right)^{2}} .
$$

Equation (13) is obtained by solving $D=0$, which has another solution

$$
\kappa_{2}=\frac{-\kappa_{1, \mathrm{eff}}+\sqrt{\Lambda}}{2\left(1-Z^{(0)}\right)} .
$$

The curve representing Eq. (17) smoothly connects with that of Eq. (13) at point (iii), where

$$
\kappa_{1, \mathrm{eff}}^{(\mathrm{iii})}=-\frac{1}{4}\left(N_{y}-N_{x}\right) .
$$


(a)

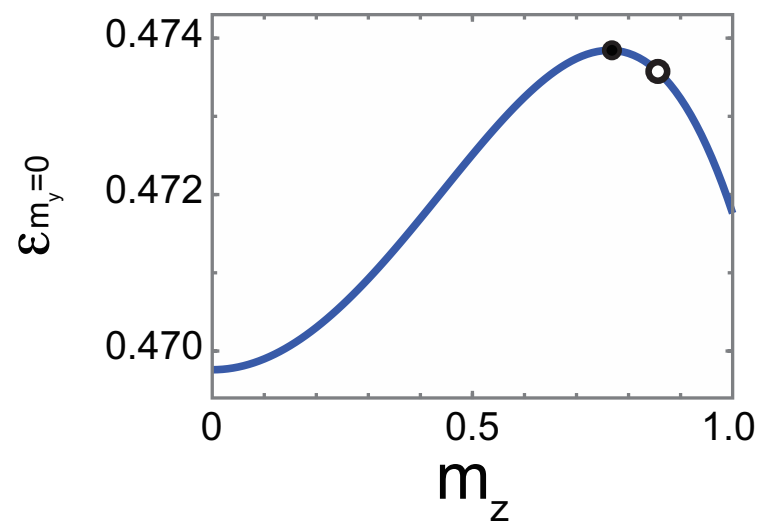

(c)

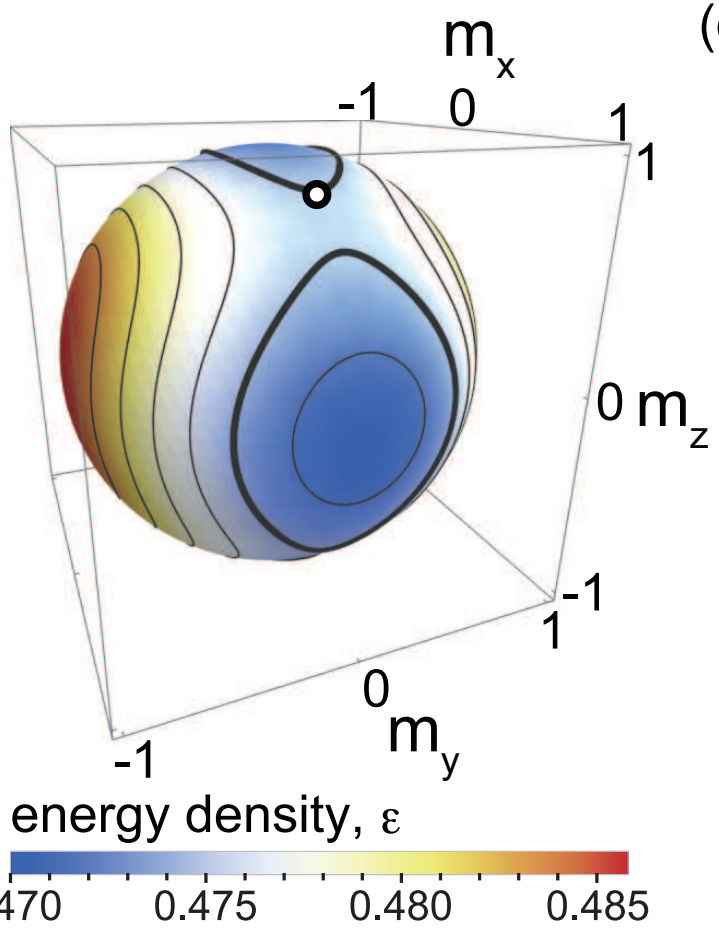

(b)

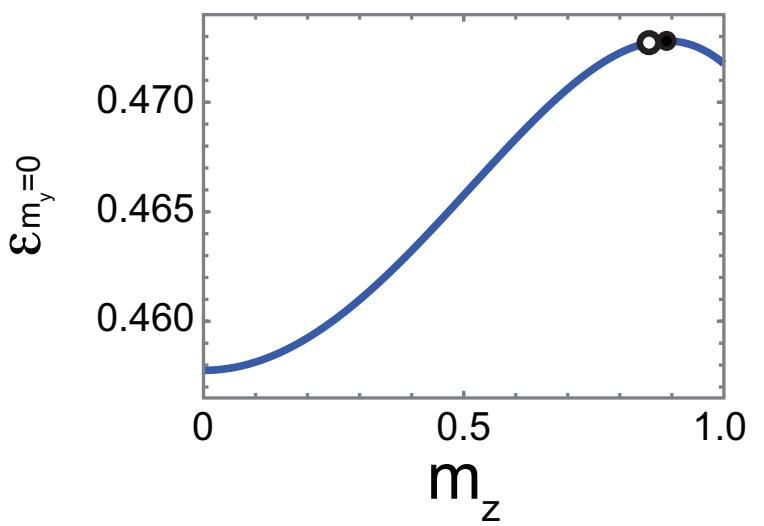

(d)

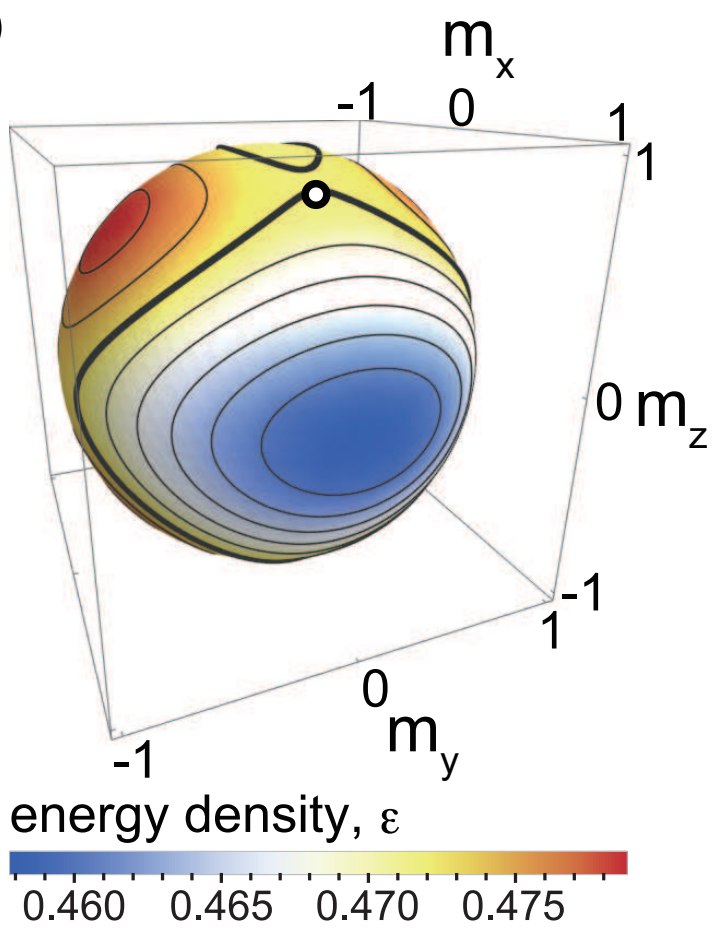

FIG. 4. (a) $m_{z}$ dependence of energy density with $m_{y}=0$. The anisotropy constants are $\left(\kappa_{1, \text { eff }}\right.$, $\left.\kappa_{2}\right)=(0.010,-0.012)$. The open and solid circles indicate the initial state, $\varepsilon_{m_{y}=0}\left(m_{z}^{(0)}\right)$, and the maximum point, respectively. (b) The same plot as (a) for the anisotropy constants of $\left(\kappa_{1, \text { eff }}\right.$, $\left.\kappa_{2}\right)=(0.010,-0.024)$. (c) Energy-density contour plot for the same parameters as in (a). The open circle indicates $\boldsymbol{m}^{(0)}$. Thick solid curves represent the contour having the same energy density as $\varepsilon\left(\boldsymbol{m}^{(0)}\right)$. (d) Energy-density contour plot for the same parameters as in (b).

In the crosshatched region, only $Z_{-}$is in $(0,1)$, and two kinds of energy-density contours coexist. One crosses the latitude with $m_{x}=0$ at $m_{z}= \pm \sqrt{Z_{-}}$and corresponds to the precession around the $m_{z}$ axis. The other corresponds to the precession around the $m_{x}$ axis. 
The boundary between the diagonally hatched region and the cross hatched region is given by

$$
\kappa_{2}=-\frac{\kappa_{1, \mathrm{eff}}}{1-Z^{(0)}}
$$

which is represented by the thick solid line in Fig. 3. This line is tangential to the curve of Eq. (17) at point (iv), where

$$
\kappa_{1, \mathrm{eff}}^{(\mathrm{iv})}=-\frac{1}{2}\left(N_{y}-N_{x}\right)
$$

Equation (19) crosses the lower boundary of Eq. (7) at point (v), where

$$
\kappa_{1, \mathrm{eff}}^{(\mathrm{v})}=\frac{\left(N_{y}-N_{x}\right)\left(1-Z^{(0)}\right)}{2 Z^{(0)}} .
$$

In the crosshatched region, the precessional switching is available if the initial state is located on the energy-density contour surrounding the $m_{x}$ axis. The condition for the precessional switching is obtained by analyzing the $m_{z}$ dependence of the energy density on the latitude with $m_{y}=0, \varepsilon_{m_{y}=0}\left(m_{z}\right)$. A typical example of $\varepsilon_{m_{y}=0}\left(m_{z}\right)$ for parameters with which the magnetization does not switch is shown in Fig. 4(a). The anisotropy constants are assumed to be $\left(\kappa_{1, \text { eff }}, \kappa_{2}\right)=(0.010,-0.012)$. The initial state, $\varepsilon_{m_{y}=0}\left(m_{z}^{(0)}\right)$, is indicated by the open circle and the maximum point of $\varepsilon_{m_{y}=0}\left(m_{z}\right)$ is indicated by the solid circle. Since the $m_{z}$ of the initial state is larger than that of the maximum point, the initial state is located on the energy-density contour surrounding the $m_{z}$ axis as shown in Fig. ए(c) and the magnetization does not switch. 


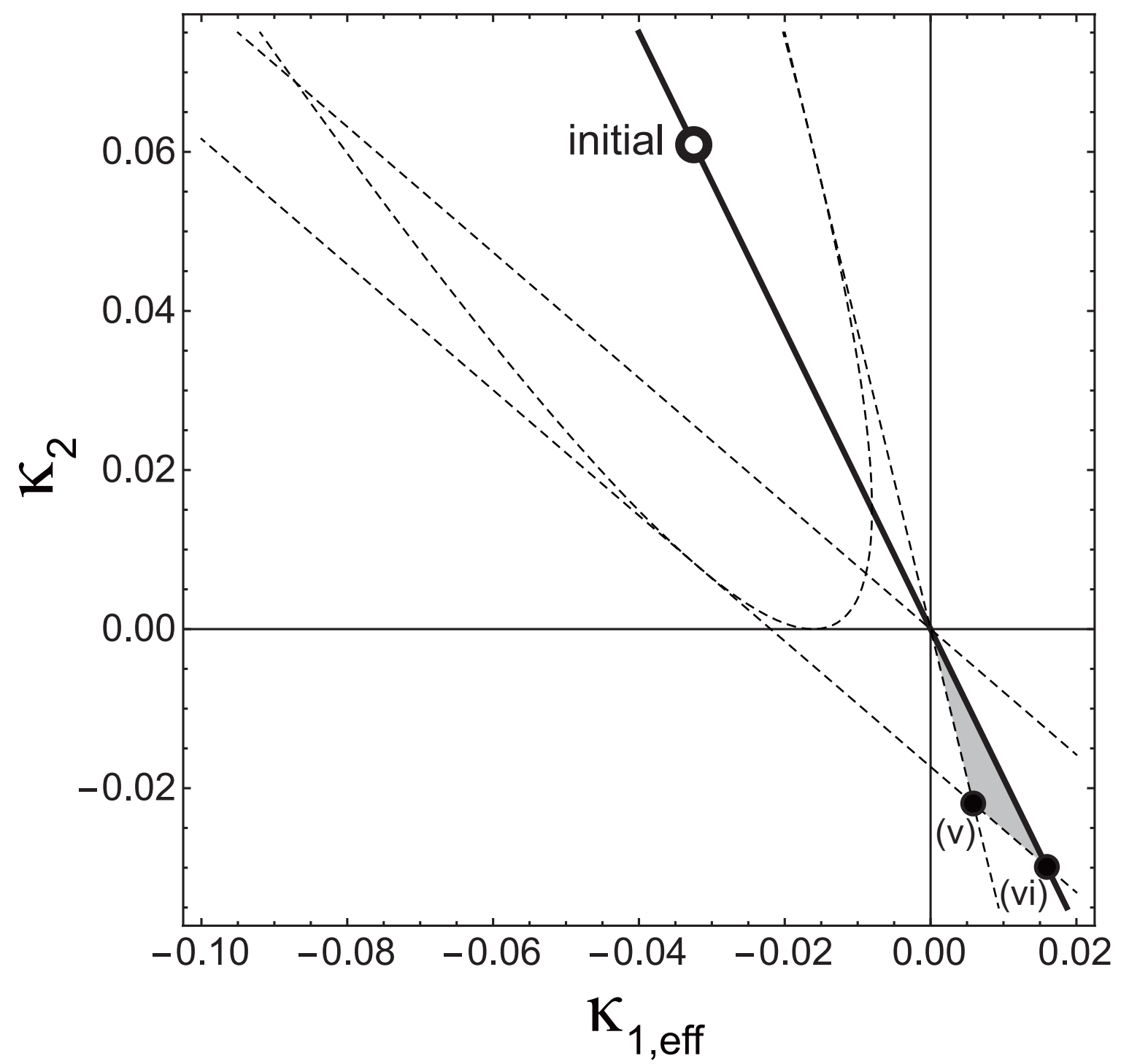

FIG. 5. The switching region in the crosshatched area in Fig. 3 is indicated by the shade triangle with vertices at the origin and at points (v) and (vi). The thin dotted lines and curves represents the boundaries shown in Fig. 3. The thick solid line represents Eq. (22). The point corresponding to the initial state is indicated by the open circle. The value of $\kappa_{1, \text { eff }}$ at the point indicated by solid circle (vi) are given in the text.

A typical example of $\varepsilon_{m_{y}=0}\left(m_{z}\right)$ for parameters with which precessional switching is available is shown in Fig. 4(b), where the anisotropy constants are assumed to be $\left(\kappa_{1, \mathrm{eff}}, \kappa_{2}\right)=(0.010,-0.024)$. Since the $m_{z}$ value of the initial state is smaller than that at the maximum point, the initial state is located on the energy-density contour surrounding the $m_{x}$ axis as shown in Fig. 4(d), and precessional switching is available. 
The boundary of the switching region is obtained by locating the initial state at the maximum point as

$$
\kappa_{2}=\frac{\kappa_{2}^{(0)}}{\kappa_{1, \mathrm{eff}}^{(0)}} \kappa_{1, \mathrm{eff}},
$$

which is indicated by the thick solid line in Fig. 5. The switching region is inside the triangle with vertices at points (v) and (vi) and the origin as shown by the shaded region in Fig. 5, The value of $\kappa_{1, \text { eff }}$ at point (vi) is given by

$$
\kappa_{1, \mathrm{eff}}^{(\mathrm{vi})}=\frac{\left(N_{y}-N_{x}\right)\left(1-Z^{(0)}\right)}{\left(Z^{(0)}\right)^{2}} .
$$




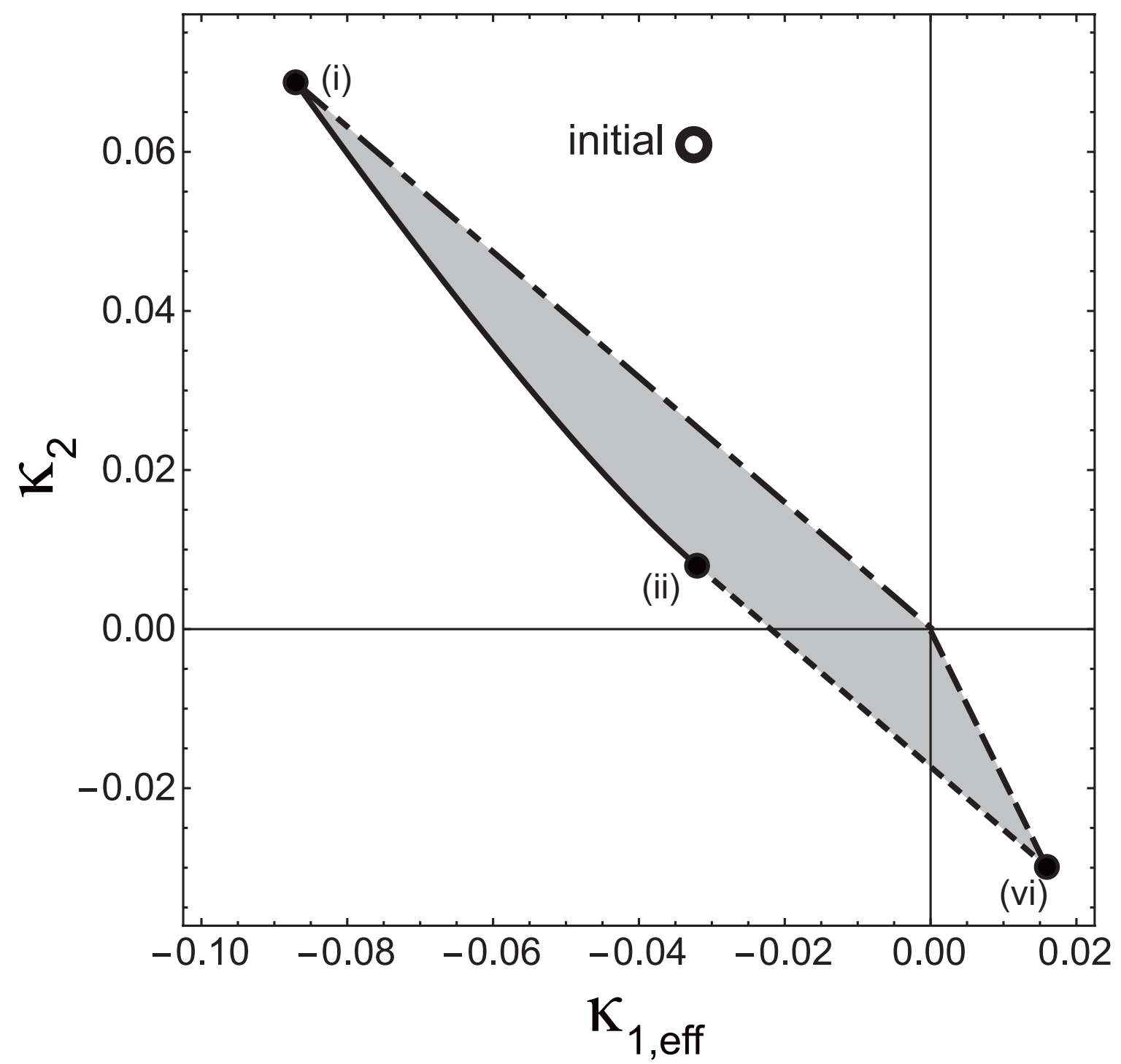

FIG. 6. The total switching region and its boundary. The initial values are indicated by the open circle. The solid circles represent the vertices of the total switching region other than the origin. The labels of the vertices are the same as those in Figs. 3 and 5 . The analytical expressions of the boundaries indicated by the solid curve, the dotted line, the dashed line and the dotted-dashed line are given in the text.

Figure [6 shows the total switching region, which is given by the combination of the vertically hatched region, the diagonally hatched region in Fig. 3 and the shaded region in Fig. 5. The boundary indicated by the solid curve is given by Eq. (13). The boundaries indicated by the dotted and dotted-dashed lines are the lower and upper boundaries of Eq. (7), respectively. The boundary indicated by the dashed line is given by Eq. (22) . 
It should be noted that as long as $N_{z} \gg N_{y}>N_{x}$ and $0<\theta^{(0)}<\pi / 2$, the values $\kappa_{1 \text {,eff }}$ at points (i), (ii), and (vi) satisfy

$$
\kappa_{1, \text { eff }}^{(\mathrm{i})}<\kappa_{1, \text { eff }}^{(\mathrm{ii})}<0<\kappa_{1, \text { eff }}^{(\mathrm{vi})}
$$

and the derived analytical expressions for the boundaries of total switching region are therefore valid.

Let us make some brief comments on the effects of the pulse width and finite temperature on switching. The pulse width which enables the precessional switching ranges from about the second quarter to the third quarter of the precession period. After turning off the voltage pulse, the magnetization relaxes to the equilibrium state with precessing around the effective field. At finite temperature, the initial state distributes around the equilibrium direction. The thermal distribution of the initial state is one of the main causes of the write error rate (WER). The WER of the voltage-controlled MRAM was studied in Refs. [12, 14] and is

known to take a minimum value at half of the precession period. Similar dependence of the WER on the pulse width is expected in our system.

\section{CONCLUSION}

In conclusion, voltage-induced magnetization dynamics in a conically magnetized free layer with an elliptic cylinder shape is studied theoretically in this paper. It is shown that precessional switching of magnetization can be performed by applying a voltage pulse even at zero-bias magnetic field. The analytical expressions of the conditions for precessional switching are derived, which is valid as long as the conically magnetized free layer is microfabricated into an elliptic cylinder shape. The results provide a practical guide for designing a bias-field-free voltage-controlled MRAM, which simplifies the device structure and reduces the fabrication cost.

\section{ACKNOWLEDGMENTS}

This work was partly supported by the ImPACT Program of the Council for Science, Technology and Innovation, and JSPS KAKENHI Grant No. JP16K17509. 


\section{Appendix A: TRAJECTORY OF PRECESSIONAL SWITCHING}

Our analysis is based on the assumption that the trajectory of precessional switching is well represented by an energy-density contour including $\boldsymbol{m}^{(0)}$ on the Bloch sphere. In order to show the validity of this assumption, we perform numerical simulations to calculate the exact trajectory by solving the Landau-Lifshitz-Gilbert (LLG) equation [28],

$$
\frac{d \boldsymbol{m}}{d t}=-\gamma_{0} \boldsymbol{m} \times \boldsymbol{H}_{\mathrm{eff}}+\alpha \boldsymbol{m} \times \frac{d \boldsymbol{m}}{d t},
$$

where $t$ is the time, $\gamma_{0}$ is the gyromagnetic ratio, and $\alpha$ is the Gilbert damping constant. The effective magnetic field, $\boldsymbol{H}_{\text {eff }}$, is defined as

$$
\boldsymbol{H}_{\mathrm{eff}}=-\frac{1}{\mu_{0} M_{\mathrm{s}}} \nabla \mathcal{E}
$$

Figure 7(a) shows the simulated trajectory (the blue curve) together with the corresponding energy-density contour (the gray curve) on the Bloch sphere. The open circle indicates the initial state, $\boldsymbol{m}^{(0)}$. The parameters are the same as in Fig. 2(c) and the damping constant is assumed to be $\alpha=0.005$. One can see that within the initial half period of precession, $0 \leq t \leq 0.56 \mathrm{~ns}$, the difference between the simulated trajectory and the corresponding energy-density contour is negligible. The scalar product between these two curves

is plotted in Fig. 7(b) as a function of $m_{z}$, which is not less than 99\%. These results strongly support the validity of our analysis. 

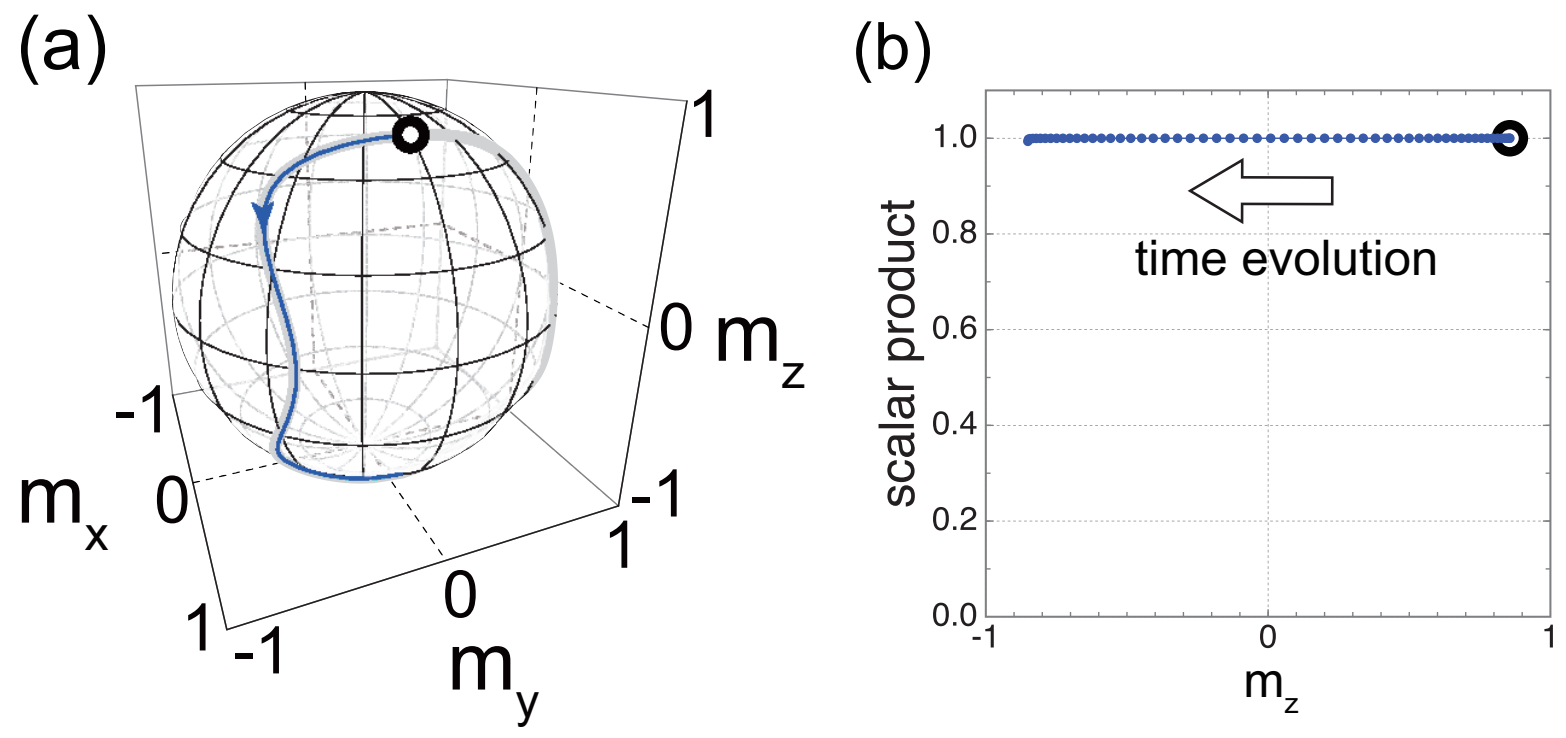

FIG. 7. Time evolution of $\boldsymbol{m}$ under voltage during precessional switching. The parameters are the same as in Fig. 2(c). (a) The simulated trajectory of $\boldsymbol{m}=\left(m_{x}, m_{y}, m_{z}\right)$ is plotted by the thin blue curve together with the corresponding energy-density contour (thick gray curve). The direction of the precession is indicated by the arrow. The open circle indicates $\boldsymbol{m}^{(0)}$. (b) The scalar product between magnetization unit vectors of the simulated trajectory and the corresponding energydensity contour is plotted as a function of $m_{z}$. The direction of the time evolution is indicated by the arrow.

[1] Martin Weisheit, Sebastian Fähler, Alain Marty, Yves Souche, Christiane Poinsignon, and Dominique Givord, "Electric field-induced modification of magnetism in thin-film ferromagnets," Science 315, 349-351 (2007).

[2] T. Maruyama, Y. Shiota, T. Nozaki, K. Ohta, N. Toda, M. Mizuguchi, A. A. Tulapurkar, T. Shinjo, M. Shiraishi, S. Mizukami, Y. Ando, and Y. Suzuki, "Large voltage-induced magnetic anisotropy change in a few atomic layers of iron," Nat. Nano. 4, 158-161 (2009).

[3] Chun-Gang Duan, Julian P. Velev, R. F. Sabirianov, Ziqiang Zhu, Junhao Chu, S. S. Jaswal, and E. Y. Tsymbal, "Surface magnetoelectric effect in ferromagnetic metal films," Phys. Rev. Lett. 101, 137201 (2008),

[4] Kohji Nakamura, Riki Shimabukuro, Yuji Fujiwara, Toru Akiyama, Tomonori Ito, and A. J. 
Freeman, "Giant modification of the magnetocrystalline anisotropy in transition-metal monolayers by an external electric field," Phys. Rev. Lett. 102, 187201 (2009).

[5] Masahito Tsujikawa and Tatsuki Oda, "Finite electric field effects in the large perpendicular magnetic anisotropy surface $\mathrm{Pt} / \mathrm{Fe} / \mathrm{Pt}(001)$ : A first-principles study," Phys. Rev. Lett. 102, 247203 (2009).

[6] T. Nozaki, Y. Shiota, M. Shiraishi, T. Shinjo, and Y. Suzuki, "Voltageinduced perpendicular magnetic anisotropy change in magnetic tunnel junctions," Appl. Phys. Lett. 96, 022506 (2010).

[7] Takayuki Nozaki, Anna Kozioł-Rachwał, Witold Skowroński, Vadym Zayets, Yoichi Shiota, Shingo Tamaru, Hitoshi Kubota, Akio Fukushima, Shinji Yuasa, and Yoshishige Suzuki, "Large voltage-induced changes in the perpendicular magnetic anisotropy of an MgO-based tunnel junction with an ultrathin Fe layer," Phys. Rev. Applied 5, 044006 (2016).

[8] M. Endo, S. Kanai, S. Ikeda, F. Matsukura, and H. Ohno, "Electric-field effects on thickness dependent magnetic anisotropy of sputtered $\mathrm{MgO} / \mathrm{Co}_{40} \mathrm{Fe}_{40} \mathrm{~B}_{20} / \mathrm{Ta}$ structures," Appl. Phys. Lett. 96, 212503 (2010).

[9] Yoichi Shiota, Takayuki Nozaki, Frédéric Bonell, Shinichi Murakami, Teruya Shinjo, and Yoshishige Suzuki, "Induction of coherent magnetization switching in a few atomic layers of FeCo using voltage pulses," Nat. Mater. 11, 39-43 (2012).

[10] Yoichi Shiota, Shinji Miwa, Takayuki Nozaki, Frédéric Bonell, Norikazu Mizuochi, Teruya Shinjo, Hitoshi Kubota, Shinji Yuasa, and Yoshishige Suzuki, "Pulse voltage-induced dynamic magnetization switching in magnetic tunneling junctions with high resistance-area product," Appl. Phys. Lett. 101, 102406 (2012).

[11] S. Kanai, M. Yamanouchi, S. Ikeda, Y. Nakatani, F. Matsukura, and H. Ohno, "Electric fieldinduced magnetization reversal in a perpendicular-anisotropy CoFeB-MgO magnetic tunnel junction," Appl. Phys. Lett. 101, 122403 (2012).

[12] Yoichi Shiota, Takayuki Nozaki, Shingo Tamaru, Kay Yakushiji, Hitoshi Kubota, Akio Fukushima, Shinji Yuasa, and Yoshishige Suzuki, "Evaluation of write error rate for voltagedriven dynamic magnetization switching in magnetic tunnel junctions with perpendicular magnetization," Appl. Phys. Express 9, 013001 (2016).

[13] C. Grezes, F. Ebrahimi, J. G. Alzate, X. Cai, J. A. Katine, J. Langer, B. Ocker, P. Khalili Amiri, and K. L. Wang, "Ultra-low switching energy and scaling in electric- 
field-controlled nanoscale magnetic tunnel junctions with high resistance-area product," Appl. Phys. Lett. 108, 012403 (2016).

[14] Yoichi Shiota, Takayuki Nozaki, Shingo Tamaru, Kay Yakushiji, Hitoshi Kubota, Akio Fukushima, Shinji Yuasa, and Yoshishige Suzuki, "Reduction in write error rate of voltage-driven dynamic magnetization switching by improving thermal stability factor," Appl. Phys. Lett. 111, 022408 (2017).

[15] J. C. Slonczewski, "Current-driven excitation of magnetic multilayers," J. Magn. Magn. Mater. 159, L1-L7 (1996).

[16] L. Berger, "Emission of spin waves by a magnetic multilayer traversed by a current," Phys. Rev. B 54, 9353-9358 (1996).

[17] E. B. Myers, D. C. Ralph, J. A. Katine, R. N. Louie, and R. A. Buhrman, "Current-induced switching of domains in magnetic multilayer devices," Science 285, 867-870 (1999).

[18] H.B.G. Casimir, J. Smit, U. Enz, J.F. Fast, H.P.J. Wijn, E.W. Gorter, A.J.W. Duyvesteyn, J.D. Fast, and J.J. de Jong, "Rapport sur quelques recherches dans le domaine du magnétisme aux laboratoires philips," J. Phys. Radium 20, 360-373 (1959).

[19] D. Apalkov and W.H. Butler, "US patent 8,780,665," US Patent 8,780,665 (2014).

[20] Rie Matsumoto, Hiroko Arai, Shinji Yuasa, and Hiroshi Imamura, "Spin-transfertorque switching in a spin-valve nanopillar with a conically magnetized free layer," Appl. Phys. Express 8, 063007 (2015).

[21] Rie Matsumoto, Hiroko Arai, Shinji Yuasa, and Hiroshi Imamura, "Theoretical analysis of thermally activated spin-transfer-torque switching in a conically magnetized nanomagnet," Phys. Rev. B 92, 140409 (2015).

[22] Jeong-Won Lee, Jong-Ryul Jeong, Sung-Chul Shin, Jonggeol Kim, and Sang-Koog Kim, "Spin-reorientation transitions in ultrathin Co films on $\operatorname{Pt}(111)$ and $\operatorname{Pd}(111)$ single-crystal substrates," Phys. Rev. B 66, 172409 (2002).

[23] Holger Stillrich, Christian Menk, Robert Fromter, and Hans Peter Oepen, "Magnetic anisotropy and the cone state in Co/Pt multilayer films," J. Appl. Phys. 105, 07C308 (2009).

[24] Kyung-Woong Park, June-Young Park, Seung-heon Chris Baek, Dae-Hoon Kim, Soo-Man Seo, Sung-Woong Chung, and Byong-Guk Park, "Electric field control of magnetic anisotropy in the easy cone state of Ta/Pt/CoFeB/MgO structures," Appl. Phys. Lett. 109, 012405 (2016).

[25] J. M. Shaw, H. T. Nembach, M. Weiler, T. J. Silva, M. Schoen, J. Z. Sun, and D. C. 
Worledge, "Perpendicular magnetic anisotropy and easy cone state in $\mathrm{Ta} / \mathrm{Co}_{60} \mathrm{Fe}_{20} \mathrm{~B}_{20} / \mathrm{MgO}$," IEEE Magnetics Letters 6, 3500404 (2015).

[26] Yu Fu, I. Barsukov, Jing Li, A. M. Gonçalves, C. C. Kuo, M. Farle, and I. N. Krivorotov, "Temperature dependence of perpendicular magnetic anisotropy in CoFeB thin films," Appl. Phys. Lett. 108, 142403 (2016).

[27] A. A. Timopheev, R. Sousa, M. Chshiev, H. T. Nguyen, and B. Dieny, "Second order anisotropy contribution in perpendicular magnetic tunnel junctions," Sci. Rep. 6, 26877 (2016)

[28] Mark D. Stiles and Jacques Miltat, "Spin-transfer torque and dynamics," in Spin Dynamics in Confined Magnetic Structures III, Topics in Applied Physics, Vol. 101, edited by Burkard Hillebrands and André Thiaville (Springer Berlin Heidelberg, 2006) pp. 225-308.

[29] R. Matsumoto and H. Imamura, "Critical current density of a spin-torque oscillator with an in-plane magnetized free layer and an out-of-plane magnetized polarizer," AIP Advances 6, 125033 (2016).

[30] M. Beleggia, M. De Graef, Y. T. Millev, D. A. Goode, and G. Rowlands, "Demagnetization factors for elliptic cylinders," J. Phys. D: Appl. Phys. 38, 3333 (2005).

[31] Shun Kanai, Martin Gajek, D. C. Worledge, Fumihiro Matsukura, and Hideo Ohno, "Electric field-induced ferromagnetic resonance in a $\mathrm{CoFeB} / \mathrm{MgO}$ magnetic tunnel junction under dc bias voltages," Appl. Phys. Lett. 105, 242409 (2014).

[32] A. Okada, S. Kanai, M. Yamanouchi, S. Ikeda, F. Matsukura, and H. Ohno, "Electric-field effects on magnetic anisotropy and damping constant in $\mathrm{Ta} / \mathrm{CoFeB} / \mathrm{MgO}$ investigated by ferromagnetic resonance," Appl. Phys. Lett. 105, 052415 (2014).

[33] Takayuki Nozaki, Hiroko Arai, Kay Yakushiji, Shingo Tamaru, Hitoshi Kubota, Hiroshi Imamura, Akio Fukushima, and Shinji Yuasa, "Magnetization switching assisted by highfrequency-voltage-induced ferromagnetic resonance," Appl. Phys. Express 7, 073002 (2014).

[34] Witold Skowroński, Takayuki Nozaki, Yoichi Shiota, Shingo Tamaru, Yakushiji Kay, Hitoshi Kubota, Akio Fukushima, Shinji Yuasa, and Yoshishige Suzuki, "Perpendicular magnetic anisotropy of $\mathrm{Ir} / \mathrm{CoFeB} / \mathrm{MgO}$ trilayer system tuned by electric fields," Appl. Phys. Express 8, 053003 (2015).

[35] Xiang Li, Kevin Fitzell, Di Wu, C. Ty Karaba, Abraham Buditama, Guoqiang Yu, 
Kin L. Wong, Nicholas Altieri, Cecile Grezes, Nicholas Kioussis, Sarah Tolbert, Zongzhi Zhang, Jane P. Chang, Pedram Khalili Amiri, and Kang L. Wang, "Enhancement of voltage-controlled magnetic anisotropy through precise control of $\mathrm{Mg}$ insertion thickness at CoFeB|MgO interface," Appl. Phys. Lett. 110, 052401 (2017). 